Vasile Ene, Quellenstrasse 18, 63571 Gelnhausen, Germany, e-mail:

gabrielaene@hotmail.com ${ }^{\dagger}$

\title{
THOMSON'S VARIATIONAL MEASURE AND NONABSOLUTELY CONVERGENT INTEGRALS
}

\begin{abstract}
In 1987 Jarník and Kurzweil [11] proved the following result: $A$ function $F:[a, b] \rightarrow \mathbb{R}$ is $A C^{*} G$ on $[a, b]$ if and only if $\mu_{F}^{*}$ (Thomson's variational measure) is absolutely continuous on $[a, b]$ and $F$ is derivable a.e. on $[a, b]$. But condition " $F$ is derivable a.e. on $[a, b]$ " is superfluous, as it was shown in [3]. In this paper we shall improve this result (from where we obtain an answer to a question of Faure [9]). Then using Faure's definition for a Kurzweil-Henstock-Stieltjes integral with respect to a function $\omega$, we give corresponding definitions for: a Denjoy*-Stieltjes integral with respect to $\omega$, a Ward-Perron-Stieltjes integral with respect to $\omega$, a Henstock-Stieltjes variational integral with respect to $\omega$, and we show that the four integrals are equivalent.
\end{abstract}

\section{Introduction}

Throughout the paper we shall use Thomson's variational measure $\mu_{F}^{*}$ for a function $F$ (see Definition 2.4). $656)$ :

In 1987, Jarník and Kurzweil proved the following result [11] (see 3.19, p.

Theorem A. A function $F:[a, b] \rightarrow \mathbb{R}$ is $A C^{*} G$ on $[a, b]$ if and only if $\mu_{F}^{*}$ is absolutely continuous and $F$ is derivable a.e. on $[a, b]$.

Key Words: Thomson's variational measure, $V B^{*}, V B, V B^{*} G, A C^{*}, V B^{*} G$, the Kurzweil-Henstock-Stieltjes integral, the Denjoy*-Stieltjes integral.

Mathematical Reviews subject classification: 26A45, 26A39, 26A46, 26A24

Received by the editors January 5, 1999

* The author died on November 11, 1998; see Real Anal. Exch. 241 (1998/99), 3. See also www.vasile-ene.subdomain.de

†The author's widow wishes to thank the referee for the so many useful comments and suggestions that have led to a shorter and improved version of this paper. 
Almost three years later, P. Y. Lee proved the same theorem [13] (see Theorem 4, p. 757), without any reference to the paper of Jarník and Kurzweil. A variant of Theorem A is presented by W. F. Pfeffer in [15] (see Theorem 6.4.4, p. 115), and he mentioned neither Jarník and Kurzweil's theorem, nor P. Y. Lee's result. Not knowing the paper of Jarník and Kurzweil, in 1994 [3], we improved Theorem A (giving credit to P. Y. Lee), showing that the condition " $F$ is derivable a.e. on $[a, b]$ " is superfluous:

Theorem B. ([3], Corollary 1, (i), (vii) or [4], Corollary 2.27.1, (i), (vii)). A function $F:[a, b] \rightarrow \mathbb{R}$ is $A C^{*} G$ on $[a, b]$ if and only if $\mu_{F}^{*}$ is absolutely continuous.

In proving Theorem B, otherwise than Jarník and Kurzweil, P. Y. Lee and W. F. Pfeffer, we haven't used the Kurzweil-Henstock theory. In 1996, using the Kurzweil-Henstock theory, Bongiorno, Di Piazza and Skvortsov also proved Theorem B without mentioning [3] (see Theorems 3 and 4 of [1]).

Using Theorem B and a result of Thomson (see Theorem 3.1), we can easily deduce the following theorem:

Theorem C. Let $F:[a, b] \rightarrow \mathbb{R}$ be a function such that $\mu_{F}^{*}$ is absolutely continuous. Then $\mu_{F}^{*}$ is $\sigma$-finite on $[a, b]$.

In this paper we shall improve Theorem B and Theorem C (see Theorem 5.1 and Theorem 3.2), and then use these results to answer to a question of Faure [9]. In performing this task we shall use many definitions and results of Faure's paper [9].

Using Faure's definition for a Kurzweil-Henstock-Stieltjes integral with respect to a function $\omega$, we give corresponding definitions for: a Denjoy*-Stieltjes integral with respect to $\omega$, a Ward-Perron-Stieltjes integral with respect to $\omega$, a Henstock-Stieltjes variational integral with respect to $\omega$, and we show that the four integrals are equivalent.

\section{Notations, Definitions and Preliminary Results}

We denote by $m^{*}(X)$ the outer measure of the set $X$ and by $m(A)$ the Lebesgue measure of $A$, whenever $A \subseteq \mathbb{R}$ is Lebesgue measurable. For the definitions of $V B, V B^{*}$ and $A C^{*}$, see [16]. Let $\langle x, y\rangle$ denote the closed interval with the endpoints $x$ and $y$. We denote by $\mathcal{P}(E)=\{X: X \subseteq E\}$ whenever $E \subseteq \mathbb{R}$. Let $C[a, b]=\{F:[a, b] \rightarrow \mathbb{R}: F$ is continuous on $[a, b]\}$ and $\operatorname{Bor}(X)=\{A \subset X: A$ is a Borel set $\}$. We denote by $\mathcal{O}(F ; X)$ the oscillation of the function $F$ on the set $X$. Let $C_{f}$ denote the set of continuity points of the function $f$. 
Definition 2.1. Let $F:[a, b] \rightarrow \mathbb{R}$, and let $\mathrm{P}$ be a closed subset of $[\mathrm{a}, \mathrm{b}]$, $c=\inf (P), d=\sup (P)$. Let $F_{P}:[c, d] \rightarrow \mathbb{R}$ be defined as follows: $F_{P}(x)=$ $F(x), x \in P$ and $F_{P}$ is linear on each $\left[c_{k}, d_{k}\right]$, where $\left\{\left(c_{k}, d_{k}\right)\right\}_{k \geq 1}$ are the intervals contiguous to $P$.

Definition 2.2. ([17]). A sequence $\left\{E_{n}\right\}$ of sets whose union is $E$ is called an $E$-form with parts $E_{n}$. If, in addition, each part $E_{n}$ is closed in $E$ (i.e. $E_{n}=\bar{E}_{n} \cap E$ ) then the $E$-form is said to be closed. An expanding $E$-form is called an $E$-chain.

Definition 2.3. Let $f:[a, b] \rightarrow \mathbb{R}$ and $E \subseteq[a, b]$. $f$ is said to be $V B^{*} G$ (respectively $A C^{*} G$ ) on $E$ if there is an $E$-form $\left\{E_{n}\right\}$ such that $f$ is $V B^{*}$ (respectively $A C^{*}$ ) on each $E_{n}$. Note that $A C^{*} G$ here differs from the definitions given in [16], because $f$ is not supposed to be continuous.

Definition 2.4. Let $E \subset \mathbb{R}, \delta: E \rightarrow(0,+\infty)$,

$$
\beta^{*}(E ; \delta)=\{(\langle x, y\rangle, x): x \in E, y \in(x-\delta(x), x+\delta(x))\} .
$$

The finite set $\pi=\left\{\left(\left\langle x_{i}, y_{i}\right\rangle, x_{i}\right)\right\}_{i=1}^{n} \subset \beta^{*}(E ; \delta)$ is said to be a partition if the $\left\{\left\langle x_{i}, y_{i}\right\rangle\right\}_{i=1}^{n}$ is a set of nonoverlapping closed intervals. Let $f: \mathbb{R} \rightarrow \mathbb{R}$,

$$
V_{\delta}^{*}(f ; E)=\sup \left\{\sum_{(\langle x, y\rangle, x) \in \pi}|f(y)-f(x)|: \pi \subset \beta^{*}(E ; \delta) \text { is a partition }\right\},
$$

and

$$
\mu_{f}^{*}(E)=\inf _{\delta} V_{\delta}^{*}(f ; E) .
$$

Note that this $\mu_{f}^{*}$ is the same as that of Thomson [19, p. 186], and it is also identical with Thomson's $\mathcal{S}_{o^{-}} \mu_{F}$ of [18] and Faure's $m_{F}[9]$.

Definition 2.5. Let $X$ be a nonempty set and $\mathcal{P}(X)=\{E: E \subseteq X\}$. Let $\alpha: \mathcal{P}(X) \rightarrow[0,+\infty]$ be a set function with $\alpha(\emptyset)=0 . \alpha$ is said to be $\sigma$-finite on $E$ if there exists a sequence $\left\{E_{i}\right\}_{i}$ of sets such that $E \subset \cup_{i} E_{i}$ and $\alpha\left(E_{i}\right) \neq+\infty$ for each $i$.

Definition 2.6. A function $\alpha: \mathcal{P}(E) \rightarrow \overline{\mathbb{R}}$ is said to be absolutely continuous on $E \subseteq \mathbb{R}$ if $\alpha(Z)=0$ whenever $Z \subseteq E$ and $m^{*}(Z)=0$.

Definition 2.7. [9] Let $F, \omega:[a, b] \rightarrow \mathbb{R}, \omega \in V B^{*} G$ and $\omega \in C[a, b]$.

- $F$ is called $\omega$-Lipschitzian on a set $E \subset[a, b]$ or $L Z_{\omega}$ on $E$, if there exists $C>0$ such that $\mu_{F}^{*}(A) \leq C \cdot \mu_{\omega}^{*}(A)$ for every subset $A \subseteq E$. The function $F$ is called generalized $\omega$-Lipschitzian or $L Z_{\omega} G$, if there exists an $[a, b]$-form $\left\{E_{n}\right\}$ such that $F$ is $\omega$-Lipschitzian on each $E_{n}$. 
- Similarly, $F$ is called $\omega$-absolutely continuous on a set $E$, or $A C_{\omega}$ on $E$, if for any $\epsilon>0$ there exists $\delta>0$ such that $A \subseteq E$ and $\mu_{\omega}^{*}(A)<\delta$ imply $\mu_{F}^{*}(A)<\epsilon$. And it is called generalized $\omega$-absolutely continuous, or $A C_{\omega} G$, if there exists an $[a, b]$-form $\left\{E_{n}\right\}$ such that $F$ is $\omega$-absolutely continuous on each $E_{n}$. If in addition each set $E_{n}$ is closed then we say that $F \in\left[A C_{\omega} G\right]$.

- One says that $F$ is $\omega$-variational normal or shortly $\omega$-normal, if $\mu_{\omega}^{*}(A)=$ 0 implies $\mu_{F}^{*}(A)=0$.

Definition 2.8. Let $\mu$ be a positive measure defined on a $\sigma$-algebra $\mathcal{A}$ of $X$. A real measure $\nu$ defined on $\mathcal{A}$ is absolutely continuous with respect to $\mu$ (shortly $\nu \ll \mu$ ) if $\nu(A)=0$ whenever $\mu(A)=0$ and $A \in \mathcal{A}$.

Remark 2.1. If $F$ is $\omega$-normal then the restrictions of the outer measures $\mu_{F}^{*}$ and $\mu_{\omega}^{*}$ on a $\sigma$-algebra $\mathcal{A}$ satisfy $\mu_{F}^{*} \ll \mu_{\omega}^{*}$.

Proposition 2.1. [16, p. 31] If $\mu$ is a positive measure on a $\sigma$-algebra $\mathcal{A}$ of $X$ and $\nu$ is a finite positive measure on $\mathcal{A}$ then $\nu \ll \mu$ if and only if for $\epsilon>0$ there is a $\delta>0$ such that $\nu(A)<\epsilon$ whenever $\mu(A)<\delta$.

\section{An Extension of Theorem C}

Lemma 3.1. Let $F:[a, b] \rightarrow \mathbb{R}, Q \subset[a, b]$ a compact set and $\mu^{*}: \mathcal{P}(Q) \rightarrow$ $[0,+\infty]$ an outer measure such that for every compact subset $S$ of $Q$, there exists a $G_{\delta}$-set $Z \subset S$ with $\bar{Z}=S$ and $\mu^{*}(Z)=0$. Then the following assertions are equivalent:

(i) $F \in V B^{*} G$ on $Q$;

(ii) each closed subset $S$ of $Q$ contains a portion on which $F \in V B^{*}$;

(iii) $F \in V B^{*} G$ on $Z$ whenever $Z$ is a $G_{\delta}$-subset of $Q$ and $\mu^{*}(Z)=0$.

Proof. (i) $\Leftrightarrow$ (ii) See Theorem 9.1 of $[16$, p. 233] ( $F$ needs not to be continuous on $Q$, because $F \in V B^{*}$ on $A \subset Q$ implies that $F \in V B^{*}$ on $\bar{A}$, see Theorem 7.1 of $[16$, p. 229]).

(i) $\Rightarrow$ (iii) This is obvious.

(iii) $\Rightarrow$ (ii) Let $S$ be a closed subset of $Q$ (so $S$ is compact). Then there is a $G_{\delta^{-}}$set $Z \subset S$, with $\bar{Z}=S$ and $\mu^{*}(Z)=0$ (see the condition on $\mu^{*}$ ). By (iii) $F \in V B^{*} G$ on $Z$, so there exists a $Z$-form $\left\{Z_{i}\right\}$ such that $F \in V B^{*}$ on each $Z_{i}$. Then $F \in V B^{*}$ on each $\bar{Z}_{i}$ (see Theorem 7.1 of $[16$, p. 229]). By 
Baire's Category Theorem [16, p. 54], there is an open interval $I$ such that $\emptyset \neq I \cap Z \subset \bar{Z}_{i_{o}}$ for some $i_{o}$. But

$$
\emptyset \neq I \cap S=I \cap \bar{Z} \subset \overline{I \cap Z} \subset \bar{Z}_{i_{o}} .
$$

Indeed, let $x \in I \cap \bar{Z}$ and let $V_{x}$ be a neighborhood of $x$. Then $I \cap V_{x}$ is a neighborhood of $x \in \bar{Z}$ too, so $V_{x} \cap I \cap Z \neq \emptyset$. Hence $x \in \overline{I \cap Z}$ and the above relation is proved. It follows that $F \in V B^{*}$ on $I \cap S$.

Lemma 3.2. (Lemma 4.2 of [9]) Let $\omega: \mathbb{R} \rightarrow \mathbb{R}, \omega \in C[a, b], \omega(x)=\omega(a)$ for $x<a, \omega(x)=\omega(b)$ for $x>b$, and $E \subset[a, b]$. If $\mu_{\omega}^{*}(E) \neq+\infty$ the function $V: \mathbb{R} \rightarrow[0,+\infty)$,

$$
V(x)= \begin{cases}0 & \text { if } x \in(-\infty, a] \\ \mu_{\omega}^{*}(E \cap[a, x]) & \text { if } x \in(a,+\infty)\end{cases}
$$

is continuous, increasing and bounded on $\mathbb{R}$.

Moreover, if $x, y \in[a, b], x<y$ then:

$V(y)-V(x)=\mu_{\omega}^{*}(E \cap[x, y])=\mu_{\omega}^{*}(E \cap(x, y))=\mu_{\omega}^{*}(E \cap[x, y))=\mu_{\omega}^{*}(E \cap(x, y])$.

Proof. That $V$ is continuous follows by Lemma 4.2 of [9], and that $V$ is increasing and bounded is evident.

Lemma 3.3. Let $\omega: \mathbb{R} \rightarrow \mathbb{R}$ be a continuous function, $\omega(x)=\omega(a)$ for $x<a$, $\omega(x)=\omega(b)$ for $x>b$, and let $S \subset[a, b]$ be a $G_{\delta}$-set with $\mu_{\omega}^{*}(S) \neq+\infty$. Then there is a null $G_{\delta}$-set $Z \subset S$ such that $\bar{Z} \supset S$ and $\mu_{\omega}^{*}(Z)=0$.

Proof. Let $d$ be the usual distance on $\mathbb{R}$ (i.e., $d(x, y)=|x-y|$ for $x, y \in$ $\mathbb{R})$. Since $(\mathbb{R}, d)$ is separable, it follows that $(S, d)$ is also a separable metric space (see for example [2, Theorem 12]). Thus there is a countable set $Z_{1}=$ $\left\{x_{1}, x_{2}, \ldots\right\} \subset S$ such that $\bar{Z}_{1} \cap S=S$. Let $V$ be the function defined in Lemma 3.2, with $E=S$. Let $j \in \mathbb{N}$. For each $x_{i}$ let $a_{j i}, b_{j i}$ be such that $x \in\left(a_{j i}, b_{j i}\right)$,

$$
V\left(b_{j i}\right)-V\left(a_{j i}\right)<\frac{1}{2^{j+i}} \quad \text { and } \quad\left(b_{j i}-a_{j i}\right)<\frac{1}{2^{j+i}}
$$

(this is possible because $V$ is continuous and increasing). Let

$$
G_{j}=S \cap\left(\cup_{i=1}^{\infty}\left(a_{j i}, b_{j i}\right)\right) \text { and } Z=\cap_{j=1}^{\infty} G_{j} .
$$


Then $Z$ is a $G_{\delta}$-subset of $S$ that contains $Z_{1}$. Hence $\bar{Z} \supset S$ and

$$
\begin{gathered}
\mu_{\omega}^{*}(Z) \leq \mu_{\omega}^{*}\left(G_{j}\right) \leq \sum_{i=1}^{\infty} \mu_{\omega}^{*}\left(\left(a_{j i}, b_{j i}\right) \cap S\right)= \\
=\sum_{i=1}^{\infty}\left(V\left(b_{j i}\right)-V\left(a_{j i}\right)\right)<\sum_{i=1}^{\infty} \frac{1}{2^{j+i}}=\frac{1}{2^{j}} \quad \text { for all } j \in \mathbb{N} .
\end{gathered}
$$

Thus $\mu_{\omega}^{*}(Z)=0$. Clearly $Z$ is a null set.

Theorem 3.1 (Thomson). [18, p. 94]. Let $F:[a, b] \rightarrow \mathbb{R}, A \subset[a, b]$. If $F$ is continuous at each point of $A$ then $F \in V B^{*} G$ on $A$ if and only if $\mu_{F}^{*}$ is $\sigma$-finite on $A$.

Lemma 3.4. Let $F:[a, b] \rightarrow \mathbb{R}, P=\bar{P} \subset[a, b], F \in V B^{*}$ on $P, F \in C[a, b]$. Then $\mu_{F}^{*}(P) \leq 2 V^{*}(F ; P)$.

Proof. We shall use Thomson's technique of [18, p. 94]. Let $A=\{x \in$ $P: x$ is an isolated point of $P$ at one side at least $\}$. By [16, p. 260], $A$ is a countable set. Since $F \in C[a, b], \mu_{F}^{*}(A)=0$. Let $\delta: P \backslash A \rightarrow(0,+\infty)$. Let $\pi=\left\{\left(\left\langle x_{i}, y_{i}\right\rangle, x_{i}\right)\right\}_{i=1}^{p} \subset \beta^{*}(P \backslash A ; \delta)$ be a partition. Split $\pi$ into

$$
\pi_{1}=\left\{\left(\left[x_{i}, y_{i}\right], x_{i}\right)\right\}_{i=1}^{m} \text { and } \pi_{2}=\left\{\left(\left[y_{i}, x_{i}\right], x_{i}\right)\right\}_{i=m+1}^{p} .
$$

In both cases we label the intervals from the left to the right. Let $c=\inf P$, $d=\sup P, y_{m}^{*} \in\left[y_{m}, d\right) \cap(P \backslash A)$ and $x_{m+1}^{*} \in\left(c, y_{m+1}\right] \cap(P \backslash A)$. Then we have

$$
\begin{gathered}
\sum_{\pi}\left|F\left(y_{i}\right)-F\left(x_{i}\right)\right| \leq \sum_{i=1}^{m-1} \mathcal{O}\left(F ;\left[x_{i}, x_{i+1}\right]\right)+\mathcal{O}\left(F ;\left[x_{m}, y_{m}^{*}\right]\right) \\
+\mathcal{O}\left(F ;\left[x_{m+1}^{*}, x_{m+1}\right]\right)+\sum_{i=m+1}^{p-1} \mathcal{O}\left(F ;\left[x_{i}, x_{i+1}\right]\right)<2 V^{*}(F ; P) .
\end{gathered}
$$

Thus $V_{\delta}^{*}(F ; P \backslash A) \leq 2 V^{*}(F ; P)$. It follows that $\mu_{F}^{*}(P) \leq \mu_{F}^{*}(P \backslash A)+\mu_{F}^{*}(A) \leq$ $2 V^{*}(F ; P)$.

Theorem 3.2 (An extension of Theorem C). Let $F:[a, b] \rightarrow \mathbb{R}$ be $\omega$-normal, where $\omega \in C[a, b]$ is a $V B^{*} G$ function. Then $F \in C[a, b]$ and $F$ is $V B^{*} G$ on $[a, b]$ (or equivalently $\mu_{F}^{*}$ is $\sigma$-finite on $[a, b]$, see Theorem 3.1). 
Proof. Since $\omega$ is continuous at $x \in[a, b]$, we have that $\mu_{\omega}^{*}(\{x\})=0$, so $F$ being $\omega$-normal, $\mu_{F}^{*}(\{x\})=0$. It follows that $F$ is continuous at $x$, so on $[a, b]$. Since $\omega$ is $V B^{*} G$ on $[a, b]$, by Theorem 7.1 of $[16$, p. 229], there exists a sequence $\left\{Q_{n}\right\}$ of compact sets such that $[a, b]=\cup_{n} Q_{n}$ and $\omega$ is $V B^{*}$ on each $Q_{n}$. By Lemma 3.4, $\mu_{\omega}^{*}\left(Q_{n}\right) \neq+\infty$. Fix some $n$ and let $S$ be a compact subset of $Q_{n}$. Then $\mu_{\omega}^{*}(S) \neq+\infty$, so by Lemma 3.3, there is a $G_{\delta}$-set $Z \subset S$, with $\bar{Z}=S$ and $\mu_{\omega}^{*}(Z)=0$. Thus $\left(\mu_{\omega}^{*}\right)_{\mid \mathcal{P}\left(Q_{n}\right)}$ satisfies the condition of Lemma 3.1. Let $Y$ be a subset of $Q_{n}$ such that $\mu_{\omega}^{*}(Y)=0$. Since $F$ is $\omega$-normal, $\mu_{F}^{*}(Y)=0$, and by Theorem 3.1, $F$ is $V B^{*} G$ on $Y$. It follows that $F$ is $V B^{*} G$ on each $Q_{n}$ (see Lemma 3.1). Hence $F$ is $V B^{*} G$ on $[a, b]$.

\section{An Answer to a Question of Faure}

Lemma 4.1 (Thomson). (A particular case of Theorem 43.1 of [18], p. 101). Let $F:[a, b] \rightarrow \mathbb{R}$ and $E \subseteq[a, b]$. Then $m^{*}(F(E)) \leq \mu_{F}^{*}(E)$.

From this lemma we obtain immediately the following corollary.

Corollary 4.1 (Faure). (Lemma 5.1 of [9]). Let $F:[a, b] \rightarrow \mathbb{R}$ and $E \subseteq[a, b]$ with $\mu_{F}^{*}(E)=0$. Then $m(F(E))=0$.

Lemma 4.2. Let $f:[a, b] \rightarrow \mathbb{R}, E \subseteq[a, b]$ and $A \subseteq\{x \in E: f$ is continuous at $x\}$. If $f \in V B^{*} G$ on $E$ then $m^{*}(f(A))=0$ if and only if $\mu_{f}^{*}(A)=0$.

Proof. Since $\mathcal{S}_{o}-\mu_{f}$ and $\mu_{f}^{*}$ are identical, the assertion follows immediately by Theorem 8 of [5] (which is an extension of Thomson's Corollary 43.4 of [18, p. 103]).

Theorem 4.1. Let $F:[a, b] \rightarrow \mathbb{R}$ and $A \subseteq[a, b]$. The following assertions are equivalent:

(i) $\mu_{F}^{*}(E)=0$;

(ii) $F$ is continuous at each point of $E, m(F(E))=0$ and $\mu_{F}^{*}(E) \neq+\infty$;

(iii) $F$ is continuous at each point of $E, m(F(E))=0$ and $\mu_{F}^{*}$ is $\sigma$-finite;

(iv) $F$ is continuous at each point of $E, m(F(E))=0$ and $F$ is $V B^{*} G$ on $E$.

Proof. (i) $\Rightarrow$ (ii) That $F$ is continuous at each point of $E$ and $\mu_{F}^{*}(E) \neq+\infty$ is obvious. By Corollary 4.1 we also have that $m(F(E))=0$.

(ii) $\Rightarrow$ (iii) This is evident.

(iii) $\Leftrightarrow$ (iv) See Theorem 3.1.

(iv) $\Rightarrow$ (i) See Lemma 4.2. 
Remark 4.1. Theorem 4.1, (i) $\Leftrightarrow$ (ii) is in fact Proposition 5.3 of Faure [9, p. 121] (our proof is different).

Example. C. A. Faure asked if in Theorem 4.1 (ii), " $\mu_{F}^{*}(E) \neq+\infty$ " can be replaced by " $F \notin V B^{*} G$ but $F$ is derivable a.e. on $E$ ". The answer is no.

Proof. Let $C$ be the Cantor ternary set. We say that $\left(a_{11}, b_{11}\right)=\left(\frac{1}{3}, \frac{2}{3}\right)$ is an open interval from the first step, $\left(a_{21}, b_{21}\right)=\left(\frac{1}{9}, \frac{2}{9}\right)$ and $\left(a_{22}, b_{22}\right)=\left(\frac{7}{9}, \frac{8}{9}\right)$ are the two intervals from the second step. In general the $2^{n-1}$ open intervals of length $\frac{1}{3^{n}}$ contiguous to $C$ are said to be the intervals from the step $n$. We denote them from the left to the right as $\left\{\left(a_{n i}, b_{n i}\right)\right\}_{i=1}^{2^{n-1}}$. Let $c_{n i}=\frac{a_{n i}+b_{n i}}{2}$ and let $\left[a_{n i}^{\prime}, b_{n i}^{\prime}\right]$ be an interval contained in $\left(a_{n i}, b_{n i}\right)$ centered in $c_{n i}$. Let $F:[0,1] \rightarrow[0,1]$,

$$
F(x)= \begin{cases}0 & \text { if } x \in C \\ \frac{1}{2^{n-1}} & \text { if } x \in \cup_{i=1}^{2^{n-1}}\left[a_{n i}^{\prime}, b_{n i}^{\prime}\right] \\ \text { linear } & \text { on }\left[a_{n i}, a_{n i}^{\prime}\right] \text { and }\left[b_{n i}^{\prime}, b_{n i}\right] .\end{cases}
$$

Then we have:

(i) $F \in C[0,1]$;

(ii) $F$ is derivable a.e. on $[0,1]$;

(iii) $F^{\prime}(x)=0$ a.e. on $E=C \cup\left(\cup_{n=1}^{\infty} \cup_{i=1}^{2^{n-1}}\left(a_{n i}^{\prime}, b_{n i}^{\prime}\right)\right)$;

(iv) $m(F(E))=0$;

(v) $F \notin V B^{*} G$ on $C$ (so on $E$ ), or equivalently (see Theorem 3.1 ), $\mu_{F}^{*}$ is not $\sigma$-finite on $C$ (so on $E$ ).

\section{An Extension of Theorem B}

Lemma 5.1. Let $F:[a, b] \rightarrow \mathbb{R}, F \in C[a, b], E \subset[a, b]$. If $\mu_{F}^{*}(E)<+\infty$ then there is a $F_{\sigma \delta}$-set $H$ such that $E \subset H$ and $\mu_{F}^{*}(H)=\mu_{F}^{*}(E)$.

Proof. For $\epsilon>0$ there is a $\delta_{\epsilon}: E \rightarrow(0,+\infty)$ such that $V_{\delta_{\epsilon}}^{*}(F ; E)<\mu_{F}^{*}(E)+$ $\epsilon / 2$. Let $E_{n}^{\epsilon}=\left\{x \in E: \delta_{\epsilon}(x)>1 / n\right\}$. Then $E=\cup_{n=1}^{\infty} E_{n}^{\epsilon}$ and $\left\{E_{n}^{\epsilon}\right\}_{n}$ is an expanding sequence of sets. Let

$$
\pi=\left\{\left(\left\langle x_{i}, y_{i}\right\rangle, x_{i}\right)\right\}_{i=1}^{p} \subset \beta\left(\overline{E_{n}^{\epsilon}} ; \frac{1}{2 n}\right) .
$$


Since $F \in C[a, b]$, for each $i$ one can choose $x_{i}^{*} \in E_{n}^{\epsilon}$ such that

$$
\left|x_{i}^{*}-x_{i}\right|<\frac{1}{2 n} \quad \text { and } \quad\left|F\left(x_{i}^{*}\right)-F\left(x_{i}\right)\right|<\frac{\epsilon}{2^{i+1}} .
$$

1) If $y_{i}<x_{i}=x_{j}<y_{j}$, then one chooses $x_{i}^{*}=x_{j}^{*} \in\left(y_{i}, y_{j}\right) \cap E_{n}^{\epsilon}$.

2) If $x_{i} \neq x_{j}$ for all $i \neq j$, then one chooses $x_{i}^{*}$ such that $\left|x_{i}^{*}-x_{i}\right|<\frac{1}{2} \delta\left(x_{i}, C_{i}\right)$ where $C_{i}=\cup_{j \neq i}\left\langle x_{j}, y_{j}\right\rangle$.

Since $\left|y_{i}-x_{i}\right|<\frac{1}{2 n}$, it follows that $\left|x_{i}^{*}-y_{i}\right|<\frac{1}{n}$, so

$$
\left(\left\langle x_{i}^{*}, y_{i}\right\rangle, x_{i}^{*}\right) \in \beta\left(E_{n}^{\epsilon} ; \frac{1}{n}\right) \subset \beta\left(E_{n}^{\epsilon} ; \delta_{\epsilon}\right) \subset \beta\left(E ; \delta_{\epsilon}\right) .
$$

We obtain that

$$
\begin{aligned}
\sum_{i=1}^{p}\left|F\left(y_{i}\right)-F\left(x_{i}\right)\right| & \leq \sum_{i=1}^{p}\left|F\left(x_{i}\right)-F\left(x_{i}^{*}\right)\right|+\sum_{i=1}^{p}\left|F\left(y_{i}\right)-F\left(x_{i}^{*}\right)\right| \\
& <\frac{\epsilon}{2}+V_{\delta_{\epsilon}}(F ; E)<\epsilon+\mu_{F}^{*}(E) .
\end{aligned}
$$

Hence

$$
\mu_{F}^{*}\left(\overline{E_{n}^{\epsilon}}\right) \leq V_{\frac{1}{2 n}}^{*}\left(F ; \overline{E_{n}^{\epsilon}}\right)<\epsilon+\mu_{F}^{*}(E) .
$$

Let $H^{\epsilon}=\cup_{n=1}^{\infty} \overline{E_{n}^{\epsilon}}$. Since any Borelian subset of $[a, b]$ is $\mu_{F}^{*}$ measurable and $\left\{\overline{E_{n}^{\epsilon}}\right\}_{n=1}^{\infty}$ is an $E$-chain (so $\left\{\overline{E_{n}^{\epsilon}}\right\}_{n=1}^{\infty}$ is an expanding sequence of sets), we have

$$
\mu_{F}^{*}\left(H^{\epsilon}\right)=\lim _{n \rightarrow \infty} \mu_{F}^{*}\left(\overline{E_{n}^{\epsilon}}\right) \leq \epsilon+\mu_{F}^{*}(E) .
$$

Let $H=\cap_{k=1}^{\infty} H^{\frac{1}{k}}$. Clearly $E \subset H$ and $H$ is of $F_{\sigma \delta}$-type. We have

$$
\mu_{F}^{*}(E) \leq \mu_{F}^{*}(H) \leq \mu_{F}^{*}\left(H^{\frac{1}{k}}\right) \leq \frac{1}{k}+\mu_{F}^{*}(E),
$$

for all $k=1,2, \ldots$. Thus $\mu_{F}^{*}(E)=\mu_{F}^{*}(H)$.

Remark 5.1. That $\mu_{F}^{*}$ in Lemma 5.1 is Borel regular was pointed out (without proof) by Thomson in $[18$, p. 43]. In fact we can prove even more, see Lemma 5.4.

Lemma 5.2. [7, Corollary 5] Let $f, g:[a, b] \rightarrow \mathbb{R}, E \subseteq[a, b]$. If $f, g \in V B^{*}$ on $E$ and $f=g$ on $E$, then

$$
\mu_{f}^{*}\left(E \cap C_{f} \cap C_{g}\right)=\mu_{g}^{*}\left(E \cap C_{f} \cap C_{g}\right) .
$$

Particularly, $\mu_{f}^{*}\left(E \cap C_{f}\right)=\mu_{\tilde{f}}^{*}\left(E \cap C_{f}\right)$, where $\tilde{f}=f_{\bar{E} \cup\{a, b\}}$ (see Definition 2.1 for the function $f_{P}$ ). 
Lemma 5.3. [7, Lemma 5] Let $f:[a, b] \rightarrow \mathbb{R}$ and $E \subseteq[a, b]$. If $f \in V B$ on $[a, b]$ then $\mu_{f}^{*}\left(E \cap C_{f}\right)=m^{*}\left(V_{f}\left(E \cap C_{f}\right)\right)$, where $\left.V_{f}(x)=V(f ;[a, x])\right)$.

Lemma 5.4. Let $F:[a, b] \rightarrow \mathbb{R}, F \in C[a, b]$. If $F$ is $V B^{*}$ on $P=\bar{P} \subset[a, b]$, then for every $E \subset P$ there is a $G_{\delta}$-set $H \subset P$ such that $\mu_{F}^{*}(E)=\mu_{F}^{*}(H)$.

Proof. Note that $\mathcal{S}_{o^{-}} \mu_{F} \equiv \mu_{F}^{*}$ and let $\tilde{F}=F_{P \cup\{a, b\}}$. By Lemma 5.2, $\mu_{F}^{*}(X)=\mu_{\tilde{F}}(X)$ for all $X \subset P$, and by Lemma $5.3, \mu_{\tilde{F}}^{*}(X)=m^{*}\left(V_{\tilde{F}}(X)\right)$ for all $X \subset[a, b]$. Thus

$$
\mu_{F}^{*}(X)=m^{*}\left(V_{\tilde{F}}(X)\right) \quad \text { for all } X \subset P .
$$

Let $G$ be a $G_{\delta^{-}}$set such that $V_{\tilde{F}}(E) \subset G$ and $m^{*}\left(V_{\tilde{F}}(E)\right)=m(G)$, and let $H=P \cap V_{\tilde{F}}^{-1}(G)$. Then $H$ is a $G_{\delta}$-set (because $V_{\tilde{F}}$ is a continuous function, so $V_{\tilde{F}}^{-1}(G)$ is a $G_{\delta}$-set). Clearly $E \subset H$ and by (1) we have

$$
\mu_{F}^{*}(E) \leq \mu_{F}^{*}(H)=m^{*}\left(V_{\tilde{F}}(H)\right) \leq m^{*}(G)=m(G)=m^{*}\left(V_{\tilde{F}}(E)\right)=\mu_{F}^{*}(E),
$$

Thus $\mu_{F}^{*}(E)=\mu_{F}^{*}(H)$.

Theorem 5.1. Let $F, \omega:[a, b] \rightarrow \mathbb{R}, \omega \in V B^{*} G$ and $\omega \in C[a, b]$. The following assertions are equivalent:

(i) $F \in L Z_{\omega} G$;

(ii) $F$ is $A C_{\omega} G$;

(iii) F is w-normal.

(iv) There is a closed $[a, b]$-form $\left\{E_{n}\right\}$ such that $\omega, F \in V B^{*}$ on each $E_{n}$ and $F$ is $A C_{\omega}$ on each $E_{n}$.

(v) There is an $[a, b]$-form $\left\{E_{n}\right\}$ with each $E_{n}$ a Borel set, such that $F$ is $L Z_{\omega}$ on each $E_{n}$.

(vi) $F \in C[a, b], F$ is $N_{\omega}$ and $F$ is $V B^{*} G$ on $[a, b]$.

Proof. (i) $\Rightarrow$ (ii) $\Rightarrow$ (iii) See Lemma 4.3 of $[9]$.

(iii) $\Rightarrow$ (iv) By Theorem 3.2, $F$ is $V B^{*} G$ on $[a, b]$, and by Theorem 7.1 of $\left[16\right.$, p. 229], there is a sequence of closed sets $\left\{E_{n}\right\}$ with $\cup_{n} E_{n}=[a, b]$ such that $\omega, F \in V B^{*}$ on each $E_{n}$. Then $\mu_{F}^{*}\left(E_{n}\right)<+\infty$ and $\mu_{\omega}^{*}\left(E_{n}\right)<+\infty$ for each $n$ (see Lemma 3.4). Since $\mu_{F \mid \mathcal{B} o r\left(E_{n}\right)}^{*}$ is a positive finite measure, by Proposition 2.1, it follows that for $\epsilon>0$ there is a $\delta=\delta\left(\epsilon, E_{n}\right)>0$ such that $\mu_{F}^{*}(A)<\epsilon$ whenever $A$ is a Borel subset of $E_{n}$ and $\mu_{\omega}^{*}(A)<\delta$. But $\left(\mu_{\omega}^{*}\right)_{\mid \mathcal{P}\left(E_{n}\right)}$ 
and $\left(\mu_{F}^{*}\right)_{\mid \mathcal{P}\left(E_{n}\right)}$ are both Borel regular (see Lemma 5.1), so for $A \subset E_{n}$ with $\mu_{\omega}^{*}(A)<\delta$, we have $\mu_{F}^{*}(A)<\epsilon$ (because there exists $A^{*} \subset \mathcal{B} \operatorname{Bor}\left(E_{n}\right)$ such that $A \subset A^{*}$ and $\left.\mu_{\omega}^{*}(A)=\mu_{\omega}^{*}\left(A^{*}\right), \mu_{F}^{*}(A)=\mu_{F}^{*}\left(A^{*}\right)\right)$. Thus $F$ is $A C_{\omega}$ on each $E_{n}$, so $F$ is $\left[A C_{\omega} G\right]$ on $[a, b]$.

(iv) $\Rightarrow(\mathrm{v})$ By Lemma 3.4 and Proposition 2.1 we have

$$
\left(\mu_{F}^{*}\right)_{\mid \mathcal{B} \text { or }\left(E_{n}\right)} \ll\left(\mu_{\omega}^{*}\right)_{\mid \mathcal{B} \text { or }\left(E_{n}\right)} .
$$

Hence, by the Radon-Nikodym Theorem, it follows that there is a Borel measurable function $f_{n}: E_{n} \rightarrow[0,+\infty)$ such that

$$
\mu_{F}^{*}(A)=\int_{A} f_{n} d \mu_{\omega}^{*}, \text { whenever } A \in \mathcal{B} \operatorname{or}\left(E_{n}\right) .
$$

Let $E_{n k}=\left\{x \in E_{n}: f_{n}(x)<k\right\}$. Then $\left\{E_{n k}\right\}_{k}$ is an $E_{n}$-chain of Borel sets. Let $A \subset E_{n k}$. Since $\left(\mu_{\omega}^{*}\right)_{\mid \mathcal{P}\left(E_{n k}\right)}$ and $\left(\mu_{F}^{*}\right)_{\mid \mathcal{P}\left(E_{n k}\right)}$ are both Borel regular (see Lemma 5.1), there exists a Borel set $A^{*} \subset E_{n k}$ such that

$$
\mu_{F}^{*}(A)=\mu_{F}^{*}\left(A^{*}\right)=\int_{A^{*}} f_{n} d \mu_{\omega}^{*} \leq k \cdot \mu_{\omega}^{*}\left(A^{*}\right)=k \cdot \mu_{\omega}^{*}(A) .
$$

(v) $\Rightarrow$ (i) This is evident.

(iii) $\Rightarrow$ (vi) Clearly $F \in C[a, b]$, and by Theorem $3.2, F$ is $V B^{*} G$ on $[a, b]$. Let $Z$ with $m(\omega(Z))=0$. By Theorem 4.1, (i), (iv), it follows that $\mu_{\omega}^{*}(Z)=0$. Since $F$ is $\omega$-normal, $\mu_{F}^{*}(Z)=0$. Again by Theorem 4.1, (i), (iv), we obtain that $m(F(Z))=0$. Thus $F \in N_{\omega}$.

(vi) $\Rightarrow$ (iii) Let $Z$ with $\mu_{\omega}^{*}(Z)=0$. By Theorem 4.1, (i), (iv), we have $m(\omega(Z))=0$. Since $F \in N_{\omega}$, it follows that $m(F(Z))=0$. Again by Theorem 4.1 , (i), (iv), we obtain that $\mu_{F}^{*}(Z)=0$, so $F$ is $\omega$-normal.

Remark 5.2. Theorem 5.1 was proved by Faure in [9, Theorem 4.7], but in (iii) $F$ is assumed to be $V B^{*} G$. As we can see from Theorem 3.2, $F$ being $V B^{*} G$ is superfluous. Also, our proof is different from that of Faure.

\section{The Equivalence of the Integrals $\mathrm{KHS}, \mathcal{D}^{*} \mathrm{~S}, \mathcal{V}$ and $\mathcal{W}$ with Respect to $\omega$}

Definition 6.1. Let $\delta:[a, b] \rightarrow(0,+\infty)$ and $E \subset[a, b]$. Let

$$
\beta_{\delta}^{o}[E]=\{([y, z] ; x): x \in E \text { and } x \in[y, z] \subset(x-\delta(x), x+\delta(x))\} .
$$

Let $\pi$ be a finite set of pairs $\left\{\left[c_{i}, d_{i}\right] ; t_{i}\right) \in \beta_{\delta}^{o}[E]$, such that $\left\{\left[c_{i}, d_{i}\right]\right\}_{i}$ is a set of nonoverlapping nondegenerate closed intervals, and let $\sigma(\pi)=\cup_{i}\left[c_{i}, d_{i}\right]$. We 
denote by $\mathcal{P}^{\circ}(E ; \delta)$ the collection of all $\pi$ defined as above. Let $f, \omega:[a, b] \rightarrow \mathbb{R}$, and let

$$
\sigma(f ; \omega ; \pi)=\sum_{i} f\left(t_{i}\right)\left(\omega\left(d_{i}\right)-\omega\left(c_{i}\right)\right), \quad S(f ; \pi)=\sum_{i}\left(f\left(d_{i}\right)-f\left(c_{i}\right)\right),
$$

for $\pi \in \mathcal{P}^{\circ}(E ; \delta)$. If $E=[a, b]$ and $\sigma(\pi)=[a, b]$ then we denote the collection of all these $\pi$ by $\mathcal{P}_{1}^{\circ}([a, b] ; \delta)$.

Remark 6.1. Recall that $D^{o}[E]=\left\{\beta_{\delta}^{o}[E]: \delta:[a, b] \rightarrow(0,+\infty)\right\}$ is called the ordinary derivation basis on the set $E$ (see for example [4, p. 87]).

Definition 6.2. [9]. Let $f, \omega:[a, b] \rightarrow \mathbb{R}$. $\mathrm{f}$ is said to be Kurzweil-HenstockStieltjes integrable (short $K H S$-integrable) on $[a, b]$ with respect to $\omega$, if there exists a real number $I$ with the following property: for $\epsilon>0$ there exists $\delta:[a, b] \rightarrow(0,+\infty)$ such that $|\sigma(f ; \omega ; \pi)-I|<\epsilon$, whenever $\pi \in \mathcal{P}_{1}^{\circ}([a, b] ; \delta)$. Then $(K H S) \int_{a}^{b} f(t) d \omega(t)=I$.

Remark 6.2. In the above definition, the real number $I$ is unique (the proof is similar to that in Remark 5.4.2 of [4]).

Definition 6.3. ([8, p. 415]) Let $\omega, F:[a, b] \rightarrow \mathbb{R}, \omega$ strictly increasing on $[a, b]$. We define the lower and upper derivatives of $F$ with respect to $\omega$ at a point $x \in[a, b]$ as follows:

$$
\underline{D}_{\omega} F(x)=\liminf _{y \rightarrow x} \frac{F(y)-F(x)}{\omega(y)-\omega(x)} \quad \text { and } \quad \bar{D}_{\omega} F(x)=\limsup _{y \rightarrow x} \frac{F(y)-F(x)}{\omega(y)-\omega(x)} .
$$

$F$ is said to be derivable with respect to $\omega$ at $x$ if $\underline{D}_{\omega} F(x)=\bar{D}_{\omega} F(x) \in \mathbb{R}$. The derivative with respect to $\omega$ of $F$ at $x$ will be their common value and will be denoted by $F_{\omega}^{\prime}(x)$.

Lemma 6.1. Let $f, \omega:[a, b] \rightarrow \mathbb{R}$ be (KHS)-integrable on $[a, b]$ with respect to $\omega$, and let $F(x)=(K H S) \int_{a}^{x} f(t) d \omega(t)$. Then $F$ is derivable with respect to $\omega$ and $F_{\omega}^{\prime}=f$ on $[a, b]$, except on a set $Z$ with $\mu_{\omega}^{*}(Z)=0$.

Proof. This is Corollary 4.8 of [9].

Lemma 6.2. Let $f, \omega:[a, b] \rightarrow \mathbb{R}$, and let $E \subset[a, b]$ with $\mu_{\omega}^{*}(E)=0$ such that $f(x)=0$ for $x \in[a, b] \backslash E$. Then $f$ is $(K H S)$-integrable with respect to $\omega$ on $[a, b]$, and its integral is 0 .

Proof. This is a particular case of Proposition 2.9 in [9]. 
Corollary 6.1. Let $f, g, \omega:[a, b] \rightarrow \mathbb{R}$. If $f$ is (KHS)-integrable with respect to $\omega$ on $[a, b]$, and $f=g$ except on a set $E$ with $\mu_{\omega}^{*}(E)=0$, then $g$ is also $(K H S)$-integrable with respect to $\omega$ on $[a, b]$ and the two integrals are equal.

Proof. The proof follows from Lemma 6.2 and the linearity of the integral.

Definition 6.4. Let $f, \omega:[a, b] \rightarrow \mathbb{R}, \omega \in V B^{*} G$ on $[a, b], \omega \in C[a, b] . f$ is said to be Denjoy*-Stieltjes integrable (short $\mathcal{D}^{*} S$-integrable) with respect to $\omega$ on $[a, b]$ if there is a $\omega$-normal function $F:[a, b] \rightarrow \mathbb{R}$ such that $F_{\omega}^{\prime}=f$ on $[a, b]$, except on a set $E$ with $\mu_{\omega}^{*}(E)=0$. We write $\left(\mathcal{D}^{*} S\right) \int_{a}^{b} f(t) d \omega(t)=$ $F(b)-F(a)$, and we say that $F$ is an indefinite $\mathcal{D}^{*} S$-integral of $f$.

Lemma 6.3. The $\mathcal{D}^{*} S$ integral is well-defined. Moreover, let $f, \omega:[a, b] \rightarrow \mathbb{R}$. If $f$ is $\left(\mathcal{D}^{*} S\right)$-integrable with respect to $\omega$ on $[a, b]$, then $f$ is $(K H S)$-integrable with respect to $\omega$ on $[a, b]$, and the two integrals are equal.

Proof. Let $F$ be an indefinite $\mathcal{D}^{*} S$ integral of $f$. Then $F_{\omega}^{\prime}=f$ on $[a, b]$ except on a set $Z$ with $\mu_{\omega}^{*}(Z)=0$. Since $F$ is $\omega$-normal, it follows that $\mu_{F}^{*}(Z)=0$. Let $f_{o}:[a, b] \rightarrow \mathbb{R}$,

$$
f_{o}(x)= \begin{cases}f(x) & \text { if } x \in[a, b] \backslash Z \\ 0 & \text { if } x \in Z\end{cases}
$$

By $\left[9\right.$, Proposition 4.5], $f_{o}$ is $(K H S)$-integrable with respect to $\omega$ on $[a, b]$, and

$$
F(x)-F(a)=(K H S) \int_{a}^{x} f_{o}(t) d \omega(t) .
$$

So the $\mathcal{D}^{*} S$ integral of $f$ is well defined. By Corollary 6.1 it follows that $f$ is $(K H S)$-integrable with respect to $\omega$ on $[a, b]$ and the two integrals are equal.

Definition 6.5. Let $f, \omega:[a, b] \rightarrow \mathbb{R}$.

- We define the following class of majorants: $\overline{\mathcal{W}}(f)=\{M:[a, b] \rightarrow \mathbb{R}:$ $M(a)=0$; there exists $\delta:[a, b] \rightarrow(0, \infty)$ such that $M(z)-M(y)>$ $f(x)(\omega(z)-\omega(y))$, whenever $x \in[y, z] \subset(x-\delta(x), x+\delta(x))\}$;

- We define the following class of minorants: $\underline{\mathcal{W}}(f)=\{m:[a, b] \rightarrow \mathbb{R}$ : $-m \in \overline{\mathcal{W}}(-f)\}$. 
- If $\overline{\mathcal{W}} \neq \emptyset$ then we denote by $\bar{J}(b)$ the lower bound of all $M(b), M \in$ $\overline{\mathcal{W}}(f)$. If $\underline{\mathcal{W}}(f) \neq \emptyset$ then we denote by $\underline{J}(b)$ the upper bound of all $m(b), m \in \underline{\mathcal{W}}(f)$.

- We say that $f$ has a $(\mathcal{W})$-integral with respect to $\omega$ on $[a, b]$, if $\overline{\mathcal{W}}(f) \times$ $\underline{\mathcal{W}}(f) \neq \emptyset$ and $\bar{J}(b)=\underline{J}(b)=(\mathcal{W}) \int_{a}^{b} f(t) d \omega(t)$.

Definition 6.6. Let $f, \omega:[a, b] \rightarrow \mathbb{R}$.

- $f$ is said to be $(\mathcal{V})$-integrable with respect to $\omega$ on $[a, b]$, if there exists $H:[a, b] \rightarrow \mathbb{R}$ such that for every $\epsilon>0$ there exist $\delta:[a, b] \rightarrow(0,+\infty)$ and $G:[a, b] \rightarrow \mathbb{R}$ with the following properties: $G(a)=0, G(b)<\epsilon, \mathrm{G}$ is increasing on $[a, b]$ and $|H(z)-H(y)-f(x)(\omega(z)-\omega(y))|<G(z)-G(y)$, whenever $x \in[y, z] \subset(x-\delta(x), x+\delta(x))$.

- $\mathrm{H}$ is called the $(\mathcal{V})$-indefinite integral of $f$ with respect to $\omega$ on $[a, b]$, and $(\mathcal{V}) \int_{a}^{b} f(t) d \omega(t)=H(b)-H(a)$.

- Clearly the $(\mathcal{V})$-integral is well defined.

Theorem 6.1. Let $f, \omega:[a, b] \rightarrow \mathbb{R}, \omega \in V B^{*} G$ and $\omega \in C[a, b]$. Then $f$ is $(K H S)$-integrable with respect to $\omega$ on $[a, b]$ if and only if $f$ is $\left(\mathcal{D}^{*} S\right)$-integrable with respect to $\omega$ on $[a, b]$ and the two integrals are equal.

Proof. " $\Rightarrow$ " The proof follows by Theorem 4.7 and Corollary 4.8 of $[9, \mathrm{p}$. 120].

"६" See Lemma 6.1.

Remark 6.3. Let $f, \omega:[a, b] \rightarrow \mathbb{R}$. The following assertions are equivalent:

- $f$ is $(K H S)$-integrable with respect to $\omega$ on $[a, b]$;

- $f$ is $\left(\mathcal{D}^{*} S\right)$-integrable with respect to $\omega$ on $[a, b]$;

- $f$ is $(\mathcal{V})$-integrable with respect to $\omega$ on $[a, b]$;

- $f$ is $(\mathcal{W})$-integrable with respect to $\omega$ on $[a, b]$;

The equivalence of the $K H S, \mathcal{W}$ and $\mathcal{V}$ integrals is known. This was proved for instance by Henstock in [10] (see Theorems 2.5.4 and 7.2.1). For the case of $K H S$ and $\mathcal{W}$ integrals this was proved as early as 1957 by Kurzweil in [12] (see Theorem 1.2.1). The equivalence of the $K H S$ and $\mathcal{D}^{*} S$ integrals follows from Theorem 6.1. 
$\mu_{\omega}^{*}$ And Nonabsolutely Convergent Integrals

\section{References}

[1] B. Bongiorno, L. Di Piazza, and V. Skvortsov, A new full descriptive characterization of Denjoy-Perron integral, Real Analysis Exchange 21 (1995-1996), no. 2, 656-663.

[2] N. Dunford and J. T. Schwartz, Linear operators, Interscience, 1958.

[3] V. Ene, Characterization of $A C^{*} G \cap \mathcal{C}, \underline{A C^{*}} \cap \mathcal{C}_{i}, A C$ and $\underline{A C}$ functions, Real Analysis Exchange 19 (1994), 491-510.

[4] V. Ene, Real functions - current topics, Lect. Notes in Math., vol. 1603, Springer-Verlag, 1995.

[5] V. Ene, Characterizations of $V B^{*} G \cap(N)$, Real Analysis Exchange 23 (1997/8), no. 2, 571-600.

[6] V. Ene, An elementary proof of the Banach-Zarecki theorem, Real Analysis Exchange 23 (1997/8), no. 1, 295-302.

[7] V. Ene, Thomson's variational measure, Real Analysis Exchange 24 (1998/9), no. 2, 523-566.

[8] C. A. Faure, Sur le théorème de Denjoy-Young-Saks, C. R. Acad. Sci. Paris 320 (1995), no. Série I, 415-418.

[9] C. A. Faure, A descriptive definition of the $\mathrm{KH}$-Stieltjes integral, Real Analysis Exchange 23 (1997-1998), no. 1, 113-124.

[10] R. Henstock, The general theory of integration, Clarendon Press, Oxford, 1991.

[11] J. Jarník and J. Kurzweil, A general form of the product integral and linear ordinary differential equations, Czech. Math. J. 37 (1987), no. 112, 642-659.

[12] J. Kurzweil, Generalized ordinary differential equations and continuous dependence on a parameter, Czech. Math. J. 7 (1957), no. 82, 418-449.

[13] P. Y. Lee, On ACG* functions, Real Analysis Exchange 15 (1989-1990), no. $2,754-760$.

[14] I. P. Natanson, Theory of functions of a real variable, 2nd. rev. ed., Ungar, New York, 1961. 
[15] W. F. Pfeffer, The Riemann approach to integration, Cambridge Univ. Press, New York, 1993.

[16] S. Saks, Theory of the integral, 2nd. rev. ed., vol. PWN, Monografie Matematyczne, Warsaw, 1937.

[17] D. N. Sarkhel, A wide Perron integral, Bull. Austral. Math. Soc. 34 (1986), 233-251.

[18] B. S. Thomson, Real functions, Lect. Notes in Math., vol. 1170, SpringerVerlag, 1985.

[19] B. S. Thomson, $\sigma$-finite Borel measures on the real line, Real Analysis Exchange 23 (1997-98), no. 1, 185-192.

[20] A. J. Ward, The Perron Stieltjes integral, Math. Zeit. 41 (1936), 578-604. 